\title{
Some Results On Distributive and Absorption Properties of Soft Operations
}

\author{
${ }^{1}$ Professor D. Singh, ${ }^{2}$ Onyeozili, I.A. (Mrs.) \\ $\left({ }^{1}\right.$ Former Professor, Indian Institute of Technology, Bombay) Mathematics DepartmentAhmadu Bello University \\ Zaria,Nigeria \\ ${ }^{2}$ Department of Mathematics University of Abuja,Nigeria (PhD Research Scholar)
}

Abstract: In this paper, we investigate some distributive and absorption properties of operations on soft sets. Keywords and Phrases: Soft sets operations, Distributivity, Absorption.

\section{Introduction}

More often than not, real life problems inherently involve uncertainties, imprecision and vagueness. In particular, such classes of problems arise in economics, engineering environmental sciences, medical sciences, etc.

In course of time, a number of mathematical theories such as probability theory, fuzzy set theory, set theory, interval mathematics theory, vague set theory, etc. formulated to solve such problems, have been found only partially successful.

The major reason for the difficulties arising with the aforesaid theories is due to the inadequacies of their parameterization tools (See Molodtsov [2]).In order to overcome these difficulties, Molodtsov [2] presented an elaborate theory of soft sets as a completely new mathematical tool with adequate parameterization for dealing with uncertainties. This prototype work of Molodtsov, besides unfolding a rich potential of soft set theory for applications, included directions for further research, especially discovering new operations on soft sets and their properties ([2], p. 21). It is encouraging to note that a number of researches in this direction have appeared during the last few years. Maji et al. [7] introduce several operations of soft sets. Ali et al. [6] and Pei and Miao [3] further introduce several other new operations as well. Based on these operations introduced in [3], [6] and [7], A. Sezgin and Alagun [1], Qin and Hong [5] and Fuli [4] establish some results concerning these new operations.

In this paper, we establish and investigate in detail new results on the distributive and absorption properties with respect to operations of union $(\tilde{\cup})$ restricted union $\left(\cup_{R}\right)$, restricted intersection (Ó) , extended intersection $\left(\cap_{\varepsilon}\right)$ and restricted difference $\left({ }_{R}\right)$ on soft sets, and provide some illustrative examples.

\section{Preliminaries}

We give some basic definitions and notions of soft set theory. Throughout this work, let $U$ be an initial universe, $E$ a set of all possible parameters with respect to $U, P(U)$ the power set of $U$ and $A \subseteq E$.

Definition 2.1 [2]

A pair $(F, A)$ is called a soft set over $U$ where $F$ is a mapping given by $F: A \rightarrow P(U)$.

For $e \in A, F(e)$ may be understood as the set of e-approximate elements of the soft set $(F, A)$. Thus

$$
(F, A)=\{F(e) \in P(U) \mid e \in A \subseteq E\} .
$$

\section{Definition 2.2 [3]}

For two soft sets $(F, A)$ and $(G, B)$ over a common universe $U$, we say that:

1. $F(A)$ is a soft subset of $(G, B)$ if

(i) $\quad A \subseteq B$

(ii) $\quad \forall e \in A, F(e) \subseteq G(e)$. We write $(F, A) \tilde{\subset}(G, B)$

2. $(F, A)$ is said to be a soft superset of $(G, B)$ if $(G, B)$ is a soft subset of $(F, A)$ denoted $(F, A) \tilde{\supset}(G, B)$. 


\section{Definition 2.3 [7]}

Two soft sets $(F, A)$ and $(G, B)$ over a common universe $U$ are said to be soft equal,denoted $(F, A)=(G, B)$, if $(F, A)$ is a soft subset of $(G, B)$ and $(G, B)$ is a soft subset of $(F, A)$.

\section{Definition 2.4 [7]}

The union of two soft sets $(F, A)$ and $(G, B)$ over a common universe $U$, denoted $(F, A) \tilde{\cup}(G, B)$ is a soft set $(\mathrm{H}, \mathrm{C})$ where $C=A \cup B$ and $\forall e \in C$,

$$
H(e)=\left\{\begin{array}{l}
F(e), \text { if } e \in A-B ; \\
G(e), \text { if } e \in B-A ; \text { and } \\
F(e) \cup G(e), \text { if } e \in A \cap B
\end{array}\right.
$$

\section{Definition $2.5[6]$}

The restricted union of two soft sets, $(F, A)$ and $(G, B)$, denoted $(F, A) \cup_{R}(G, B)$, is a soft set $(H, C)$ where $C=A \cap B \neq \varnothing$ and $\forall e \in C, H(e)=F(e) \cup G(e)$.

\section{Definition 2.6 [6]}

The extended intersection of two soft sets $(F, A)$ and $(G, B)$, denoted $(F, A) \cap_{\varepsilon}(G, B)$, is a soft set $(H, C)$ where $C=A \cup B$ and $\forall e \in C$,

$$
H(e)=\left\{\begin{array}{l}
F(e), \text { if } e \in A-B ; \\
G(e), \text { if } e \in B-A ; \text { and } \\
F(e) \cap G(e), \text { if } e \in A \cap B .
\end{array}\right.
$$

\section{Definition $2.7[6]$}

The restricted intersection of two soft sets $(F, A)$ and $(G, B)$, denoted $(F, A) \mathrm{O}(G, B)$, is a soft set $(H, C)$, where $C=A \cap B \neq \varnothing$ and $\forall e \in C$,

$$
H(e)=F(e) \cap G(e) .
$$

\section{Definition 2.8 [6]}

The restricted difference of two soft sets $(F, A)$ and $(G, B)$, denoted $(F, A){ }_{R}(G, B)$, is a soft set $(H, C)$ where $C=A \cap B \neq \varnothing$ and $\forall e \in C$,

\section{Definition 2.9 [1]}

$$
H(e)=F(e)-G(e) .
$$

The restricted symmetric difference of two soft sets $(F, A)$ and $(G, B)$ over a common universe $U$, denoted $(F, A) \bar{\Delta}(G, B)$, is a soft set defined by

\section{Theorem 2.1 [1,5]}

$$
(F, A) \tilde{\Delta}(G, B)=\left((F, A) \cup_{R}(G, B)\right)_{\smile_{R}}((F, A) \text { Ó }(G, B))
$$

Let $(F, A)$ and $(G, B)$ be two soft sets over a common universe $U$. Then the following hold:

$$
\begin{array}{ll}
(F, A) \tilde{\Delta}(G, B)=\left((F, A) \smile_{R}(G, B)\right) \cup_{R}((G, B) & (F, A)) \\
(F, A) \cup_{R}(G, B)=(G, B) \cup_{R}(F, A) . & \smile_{R}
\end{array}
$$

\section{Properties Of Operations On Soft Sets}

Theorem 3.1 (Distributive Properties)

Let $(F, A),(G, B)$ and $(H, C)$ be soft sets over a common universe. Then the following hold:

(i) $\quad(F, A) \cup_{R}((G, B) \cup(H, C))=\left((F, A) \cup_{R}(G, B)\right) \cup\left((F, A) \cup_{R}(H, C)\right)$

(ii) $\quad(F, A) \dot{O}\left((G, B) \cap_{\varepsilon}(H, C)\right)=((F, A) \dot{O}(G, B)) \cap_{\varepsilon}((F, A) \dot{O}(H, C))$

(iii) $\quad(F, A) \tilde{\cup}\left((G, B) \cup_{R}(H, C)\right) \neq((F, A) \tilde{\cup}(G, B)) \cup_{R}((F, A) \tilde{\cup}(H, C))$ 
(iv) $(F, A) \cap_{\varepsilon}((G, B)$ Ó $(H, C)) \neq\left((F, A) \cap_{\varepsilon}(G, B)\right)$ Ó $\left((F, A) \cap_{\varepsilon}(H, C)\right)$

(v) $\quad(F, A)$ Ó $\left((G, B) \smile_{R}(H, C)\right)=((F, A)$ Ó $(G, B)) \smile_{R}((F, A)$ Ó $(H, C))$

(vi) $\quad(F, A) \cup_{R}\left((G, B) \smile_{R}(H, C)\right) \neq\left((F, A) \cup_{R}(G, B)\right) \smile_{R}\left((F, A) \cup_{R}(H, C)\right)$

(vii) $\quad(F, A) \cap_{\varepsilon}\left((G, B) \smile_{R}(H, C)\right) \neq\left((F, A) \cap_{\varepsilon}(G, B)\right)\left((F, A) \cap_{\varepsilon}(H, C)\right)$

(viii) $(F, A) \tilde{\cup}\left((G, B) \smile_{\smile_{R}}^{\smile_{R}}(H, C)\right) \neq((F, A) \tilde{\cup}(G, B)) \smile_{\smile_{R}}((F, A) \tilde{\cup}(H, C))$

Proof (i): Let $(G, B) \tilde{\cup}(H, C)=(K, B \cup C)$ where $\forall \alpha \in(B \cup C)$,

$$
K(\alpha)=\left\{\begin{array}{l}
G(\alpha), \text { if } \alpha \in B-C ; \\
H(\alpha), \text { if } \alpha \in C-B ; \text { and } \\
G(\alpha) \cup H(\alpha), \text { if } \alpha \in B \cap C .
\end{array}\right.
$$

Let $(F, A) \cup_{R}(K, B \cup C)=(J, A \cap(B \cup C))$ where $\forall \alpha \in A \cap(B \cup C)$,

$$
\begin{aligned}
& J(\alpha)=F(\alpha) \cup K(\alpha) . \\
& =\left\{\begin{array}{l}
F(\alpha) \cup G(\alpha), \alpha \in A \cap(B-C) ; \\
F(\alpha) \cup H(\alpha), \alpha \in A \cap(C-B) ; \text { and } \\
F(\alpha) \cup G(\alpha) \cup H(\alpha), \alpha \in A \cap(B \cap C) .
\end{array}\right.
\end{aligned}
$$

Let $(F, A) \cup_{R}(G, B)=(L, A \cap B)$

where $\forall \alpha \in A \cap B, \quad L(\alpha)=F(\alpha) \cup G(\alpha)$.

$$
\text { Let }(F, A) \cup_{R}(H, C)=(M, A \cap B)
$$

where $\forall \alpha \in A \cap C$,

$$
M(\alpha)=F(\alpha) \cup H(\alpha) .
$$

Let $(L, A \cap B) \tilde{\cup}(M, A \cap C)=(I,(A \cap B) \cup(A \cap C)$

where $\forall \alpha \in A \cap(B \cup C)$,

$$
=(I, A \cap(B \cup C))
$$

$$
\begin{aligned}
I(\alpha) & =\left\{\begin{array}{l}
L(\alpha), \alpha \in(A \cap B)-(A \cap C) ; \\
M(\alpha), \alpha \in(A \cap C)-(A \cap B) ; \text { and } \\
L(\alpha) \cup M(\alpha), \alpha \in A \cap(B \cap C),
\end{array}\right. \\
& =\left\{\begin{array}{l}
F(\alpha) \cup G(\alpha), \alpha \in A \cap(B-C) ; \\
F(\alpha) \cup H(\alpha), \alpha \in A \cap(C-B) ; \text { and } \\
{[F(\alpha) \cup G(\alpha)] \cup[F(\alpha) \cup H(\alpha)]=F(\alpha) \cup\{G(\alpha) \cup H(\alpha)\}, \alpha \in A \cap(B \cap C) .}
\end{array}\right.
\end{aligned}
$$

Hence, $J(\alpha)=I(\alpha) \forall \alpha \in A \cap(B \cup C)$; i.e., (i) holds.

Similarly, (ii) can be proved.

(iii) Let $(G, B) \cup_{R}(H, C)=(K, B \cap C)$ where $\forall \alpha \in B \cap C$,

$$
K(\alpha)=G(\alpha) \cup H(\alpha) .
$$

Let $(F, A) \tilde{\cup}(K, B \cap C)=(I, A \cup(B \cap C))$ where $\forall \alpha \in A \cup(B \cap C)$,

$$
\begin{aligned}
I(\alpha) & =\left\{\begin{array}{l}
F(\alpha), \alpha \in A-(B \cap B) ; \\
K(\alpha), \alpha \in(B \cap C)-A ; \text { and } \\
F(\alpha) \cup K(\alpha), \alpha \in A \cap(B \cap C) .
\end{array}\right. \\
& =\left\{\begin{array}{l}
F(\alpha), \alpha \in A-(B \cap C) ; \\
G(\alpha) \cup H(\alpha), \alpha \in(B \cap C)-A ; \text { and } \\
F(\alpha) \cup\{G(\alpha) \cup H(\alpha)\}, \alpha \in A \cap(B \cap C) .
\end{array}\right.
\end{aligned}
$$

Also, let $(F, A) \tilde{\cup}(G, B)=(L, A \cup B)$ where $\forall \alpha \in A \cup B$, 


$$
L(\alpha)=\left\{\begin{array}{l}
F(\alpha), \alpha \in A-B ; \\
G(\alpha), \alpha \in B-A ; \text { and } \\
F(\alpha) \cup G(\alpha), \alpha \in A \cap B .
\end{array}\right.
$$

Let $(F, A) \tilde{\cup}(H, C)=(M, A \cup C)$ where $\forall \alpha \in A \cup C$,

$$
M(\alpha)=\left\{\begin{array}{l}
F(\alpha), \alpha \in A-C ; \\
H(\alpha), \alpha \in C-A ; \text { and } \\
F(\alpha) \cup H(\alpha), \alpha \in A \cap C .
\end{array}\right.
$$

Let $(L, A \cup B) \cup_{R}(M, A \cup C)=(N,(A \cup B) \cap(A \cup C))$ where $\forall \alpha \in A \cup(B \cap C)$,

$$
N(\alpha)=L(\alpha) \cup M(\alpha) .=\left\{\begin{array}{l}
F(\alpha), \alpha(A-B) \cap(A-C)=A-(B \cup C) ; \\
F(\alpha) \cup G(\alpha), \alpha \in(A \cap B)-C ; \\
F(\alpha) \cup H(\alpha), \alpha \in(A \cap C)-B ; \\
G(\alpha) \cup H(\alpha), \alpha \in(B \cap C)-A ; \text { and } \\
F(\alpha) \cup\{G(\alpha) \cup H(\alpha)\}, \alpha \in A \cap B \cap C .
\end{array}\right.
$$

Thus $I \neq N$. Hence (iii) holds. Similarly, (iv) can be proved.

(v) Let $(G, B) \smile_{R}(H, C)=(K, B \cap C)$ where $\forall \alpha \in B \cap C$,

$$
K(\alpha)=G(\alpha)-H(\alpha) \text {. }
$$

Let $(F, A)$ Ó $(K, B \cap C)=(L, A \cap(B \cap C))$ where $\forall \alpha \in A \cap(B \cap C)$,

$$
\begin{aligned}
L(\alpha) & =F(\alpha) \cap K(\alpha), \\
& =F(\alpha) \cap\{G(\alpha)-H(\alpha)\} .
\end{aligned}
$$

Let $(F, A)$ Ó $(G, B)=(I, A \cap B)$ where $\forall \alpha \in A \cap B \cap C$,

$$
I(\alpha)=F(\alpha) \cap G(\alpha) .
$$

Let $(F, A)$ Ó $(H, C)=(J, A \cap C)$ where $\forall \alpha \in A \cap C$,

$$
J(\alpha)=F(\alpha) \cap H(\alpha) .
$$

Let $(I, A \cap B) \smile_{R}(J, A \cap C)=(M,(A \cap B) \cap(A \cap C)$

where $\forall \alpha \in(A \cap B) \cap C$,

$$
=(M,(A \cap B) \cap C)
$$

$$
\begin{aligned}
N(\alpha) & =I(\alpha)-J(\alpha) \\
& =[F(\alpha) \cap G(\alpha)]-[F(\alpha) \cap H(\alpha)] \\
& =F(\alpha) \cap\{G(\alpha)-H(\alpha)\} .
\end{aligned}
$$

It follows, $L$ and $M$ are the same mappings on $A \cap B \cap C$.

Hence (v) hold.

(vi) Let $(G, B) \smile_{\mathbb{R}}(H, C)=(K, B \cap C)$ where $\forall \alpha \in B \cap C$,

$K(\alpha)=G(\alpha)-H(\alpha)$.

Let $(F, A) \cup_{R}(K, B \cap C)=(J, A \cap(B \cap C))$ where $\forall \alpha \in A \cap(B \cap C)$,

$$
\begin{aligned}
J(\alpha) & =F(\alpha) \cup K(\alpha) \\
& =F(\alpha) \cup\{G(\alpha)-H(\alpha)\} .
\end{aligned}
$$

Let $(F, A) \cup_{R}(G, B)=(L, A \cap B)$ where $\forall \alpha \in A \cap B$,

$L(\alpha)=F(\alpha) \cup G(\alpha)$. 
Let $(F, A) \cup_{R}(H, C)=(M, A \cap C)$ where $\forall \alpha \in A \cap C$,

$M(\alpha)=F(\alpha) \cup H(\alpha)$.

Let $(L, A \cap B) \quad(M, A \cap C)=(N,(A \cap B) \cap(A \cap C))$

where $\forall \alpha \in(\widetilde{A \cap} B) \cap(A \cap C)=A \cap(B \cap C)$,

$$
\begin{aligned}
N(\alpha) & =L(\alpha)-M(\alpha) \\
& =\{F(\alpha) \cup G(\alpha)\}-\{F(\alpha) \cup H(\alpha)\} \\
& \neq F(\alpha) \cup\{G(\alpha) \cup H(\alpha)\} .
\end{aligned}
$$

Hence $J \neq N$ on $A \cap(B \cap C)$, which completes the proof.

(vii) $\quad(G, B) \quad(H, C)=(K, B \cap C)$ where $\forall \alpha \in B \cap C$,

$K(\alpha)=\mathbb{B}(\alpha)-H(\alpha)$

Let $(F, A) \cap_{\varepsilon}(K, B \cap C)=(I, A \cup(B \cap C))$ where $\forall \alpha \in A \cup(B \cap C)$,

$$
\begin{aligned}
I(\alpha) & =\left\{\begin{array}{l}
F(\alpha), \alpha \in A-(B \cap C) ; \\
K(\alpha), \alpha \in(B \cap C)-A ; \text { and } \\
F(\alpha) \cap K(\alpha), \alpha \in A \cap(B \cap C)
\end{array}\right. \\
& =\left\{\begin{array}{l}
F(\alpha), \alpha \in A-(B \cap C) ; \\
G(\alpha)-H(\alpha), \alpha \in(B \cap C)-A ; \text { and } \\
F(\alpha) \cap\{G(\alpha)-H(\alpha)\}, \alpha \in A \cap(B \cap C) .
\end{array}\right.
\end{aligned}
$$

Let $(F, A) \cap_{\varepsilon}(G, B)=(R, A \cup B)$ where $\forall \alpha \in A \cup B$,

$$
R(\alpha)=\left\{\begin{array}{l}
F(\alpha), \alpha \in A-B ; \\
G(\alpha), \alpha \in B-A ; \text { and } \\
F(\alpha) \cap G(\alpha), \alpha \in A \cap B .
\end{array}\right.
$$

Let $(F, A) \cap_{\varepsilon}(H, C)=(L, A \cup C)$ where $\forall \alpha \in A \cup C$,

$$
L(\alpha)=\left\{\begin{array}{l}
F(\alpha), \alpha \in A-C ; \\
H(\alpha), \alpha \in C-A ; \text { and } \\
F(\alpha) \cap H(\alpha), \alpha \in A \cap C .
\end{array}\right.
$$

Let $(R, A \cup B) \quad(L, A \cup C)=(M,(A \cup B) \cap(A \cup C))$ where $\forall \alpha \in(A \cup B) \cap_{\mathrm{R}}(A \cup C)=A \cup(B \cap C), M(\alpha)=R(\alpha)-L(\alpha)$

$$
=\left\{\begin{array}{l}
\varnothing, \alpha \in A-(B \cup C) ; \\
G(\alpha)-F(\alpha), \alpha \in(A \cap B)-C ; \\
G(\alpha)-\{F(\alpha) \cap H(\alpha)\}, \alpha \in A \cap B \cap C ; \\
G \alpha)-H(\alpha), \alpha \in(B \cap C)-A ; \text { and } \\
F(\alpha)-\{F(\alpha) \cap H(\alpha)\}, \alpha \in(A \cap C)-B .
\end{array}\right.
$$

Hence mappings $I$ and $M$ are not equal on $A \cup(B \cap C)$. This completes the proof of (vii) Similarly, (viii) can be proved.

We now illustrate (ii) in the following example.

\section{Example 3.1}

Let a universe set $U$ be a set of eight different types of cars given by $U=\left\{u_{1}, u_{2}, u_{3}, u_{4}, u_{5}, u_{6}, u_{7}, u_{8}\right\}$ and let the parameter set $E$ with respect to $U$ be $E=\left\{e_{1}, e_{2}, e_{3}, e_{4}, e_{5}, e_{6}, e_{7}\right\}$, where each parameter $e_{i} i=1,2,3, \ldots, 7$ stands for fuel efficient, durable, modern, spacious, portable, luxury and flashy, respectively.

Let $A=\left\{e_{1}, e_{2}, e_{3}, e_{6}\right\}, B=\left\{e_{2}, e_{3}, e_{4}, e_{5}\right\}$ and $C=\left\{e_{3}, e_{4}, e_{6}, e_{7}\right\}$.

Let $(F, A),(G, B)$ and $(H, C)$ be three soft sets over $U$ such that 


$$
\begin{aligned}
& F\left(e_{1}\right)=\left\{u_{1}, u_{2}, u_{3}, u_{4}\right\} ; G\left(e_{2}\right)=\left\{u_{4}, u_{5}, u_{6}, u_{7}\right\} ; H\left(e_{3}\right)=\left\{u_{3}, u_{4}, u_{7}, u_{8}\right\} ; \\
& F\left(e_{2}\right)=\left\{u_{3}, u_{4}, u_{5}, u_{6}\right\} ; G\left(e_{3}\right)=\left\{u_{1}, u_{2}, u_{3}, u_{4}\right\} ; H\left(e_{4}\right)=\left\{u_{4}, u_{5}, u_{7}\right\} ; \\
& F\left(e_{3}\right)=\left\{u_{1}, u_{2}, u_{4}, u_{7}\right\} ; G\left(e_{4}\right)=\left\{u_{4}, u_{5}, u_{6}, u_{7}, u_{8}\right\} ; H\left(e_{6}\right)=\left\{u_{2}, u_{4}, u_{6}, u_{8}\right\} ; \text { and } \\
& F\left(e_{6}\right)=\left\{u_{2}, u_{3}, u_{4}\right\} ; G\left(e_{5}\right)=\left\{u_{1}, u_{2}, u_{4}, u_{7}\right\} ; H\left(e_{7}\right)=\left\{u_{2}, u_{3}, u_{5}, u_{7}\right\} .
\end{aligned}
$$

Now, let $(G, B) \cup_{R}(H, C)=(K, B \cap C)$ where $\forall \alpha \in B \cap C=\left\{e_{3}, e_{4}\right\}$, $K(\alpha)=G(\alpha) \cup H(\alpha)$.

Then,

$$
K\left(e_{3}\right)=G\left(e_{3}\right) \cup H\left(e_{3}\right)=\left\{u_{1}, u_{2}, u_{3}, u_{4}, u_{7}, u_{8}\right\}
$$

and

$$
K\left(e_{4}\right)=G\left(e_{4}\right) \cup H\left(e_{4}\right)=\left\{u_{4}, u_{5}, u_{6}, u_{7}, u_{8}\right\} .
$$

Let $(F, A) \tilde{\cup}(K, B \cap C)=(J, A \cup(B \cap C))$ where $\forall \alpha \in A \cup(B \cap C)=\left\{e_{1}, e_{2}, e_{3} . e_{4}\right\}$,

It follows,

$$
J(\alpha)=\left\{\begin{array}{l}
F(\alpha), \alpha \in A-(B \cap C)=\left\{e_{1}, e_{2}, e_{6}\right\} ; \\
K(\alpha), \alpha \in(B \cap C)-A=\left\{e_{4}\right\} ; \text { and } \\
F(\alpha) \cup K(\alpha), \alpha \in A \cap(B \cap C)=\left\{e_{3}\right\} .
\end{array}\right.
$$

$$
\begin{aligned}
& J\left(e_{1}\right)=F\left(e_{1}\right)=\left\{u_{1}, u_{2}, u_{3}, u_{4}\right\} ; \\
& J\left(e_{2}\right)=F\left(e_{2}\right)=\left\{u_{3}, u_{4}, u_{5}, u_{6}\right\} ; \\
& J\left(e_{3}\right)=F\left(e_{3}\right) \cup K\left(e_{3}\right)=\left\{u_{1}, u_{2}, u_{3}, u_{4}, u_{7}, u_{8}\right\} ; \\
& J\left(e_{4}\right)=F\left(e_{4}\right)=\left\{u_{4}, u_{5}, u_{6}, u_{7}, u_{8}\right\} ; \text { and } \\
& J\left(e_{6}\right)=F\left(e_{6}\right)=\left\{u_{2}, u_{3}, u_{4}\right\} .
\end{aligned}
$$

Also,

let $(F, A) \tilde{\cup}(G, B)=(L, A \cup B)$ where $\forall \alpha \in A \cup B=\left\{e_{1}, e_{2}, e_{3} . e_{4}, e_{5}, e_{6}\right\}$,

Thus,

$$
L(\alpha)=\left\{\begin{array}{l}
F(\alpha), \alpha \in A-B=\left\{e_{1}, e_{6}\right\} ; \\
G(\alpha), \alpha \in B-A=\left\{e_{4}, e_{5}\right\} ; \text { and } \\
F(\alpha) \cup G(\alpha), \alpha \in A \cap B=\left\{e_{2}, e_{3}\right\} .
\end{array}\right.
$$

$$
\begin{aligned}
& L\left(e_{1}\right)=F\left(e_{1}\right)=\left\{u_{1}, u_{2}, u_{3}, u_{4}\right\} ; \\
& L\left(e_{2}\right)=F\left(e_{2}\right) \cup G\left(e_{2}\right)=\left\{u_{3}, u_{4}, u_{5}, u_{6}, u_{8}\right\} ; \\
& L\left(e_{3}\right)=G\left(e_{3}\right) \cup G\left(e_{3}\right)=\left\{u_{1}, u_{2}, u_{3}, u_{4}, u_{7}\right\} ; \\
& L\left(e_{4}\right)=G\left(e_{4}\right)=\left\{u_{4}, u_{5}, u_{6}, u_{7}, u_{8}\right\} ; \\
& L\left(e_{5}\right)=F\left(e_{5}\right)=\left\{u_{2}, u_{3}, u_{4}\right\} ; \text { and } \\
& L\left(e_{6}\right)=F\left(e_{6}\right)=\left\{u_{2}, u_{3}, u_{4}\right\} .
\end{aligned}
$$

Let $(F, A) \tilde{\cup}(H, C)=(M, A \cup C)$ where $\forall \alpha \in A \cup C=\left\{e_{1}, e_{2}, e_{3} . e_{4}, e_{6}, e_{7}\right\}$,

We have

$$
M(\alpha)=\left\{\begin{array}{l}
F(\alpha), \alpha \in A-C=\left\{e_{1}, e_{2}\right\} ; \\
H(\alpha), \alpha \in C-A=\left\{e_{4}, e_{7}\right\} ; \text { and } \\
F(\alpha) \cup H(\alpha), \alpha \in A \cap C=\left\{e_{3}, e_{6}\right\} .
\end{array}\right.
$$




$$
\begin{aligned}
& M\left(e_{1}\right)=F\left(e_{1}\right)=\left\{u_{1}, u_{2}, u_{3}, u_{4}\right\} ; \\
& M\left(e_{2}\right)=F\left(e_{2}\right)=\left\{u_{3}, u_{4}, u_{5}, u_{6}\right\} ; \\
& M\left(e_{3}\right)=G\left(e_{3}\right) \cup H\left(e_{3}\right)=\left\{u_{1}, u_{2}, u_{3}, u_{4}, u_{7}, u_{8}\right\} ; \\
& M\left(e_{4}\right)=G\left(e_{4}\right)=\left\{u_{4}, u_{5}, u_{7}\right\} ; \\
& M\left(e_{6}\right)=F\left(e_{6}\right) \cup H\left(e_{6}\right)=\left\{u_{2}, u_{3}, u_{4}, u_{6}, u_{8}\right\} ; \text { and } \\
& M\left(e_{7}\right)=F\left(e_{7}\right)=\left\{u_{2}, u_{3}, u_{5}, u_{7}\right\} .
\end{aligned}
$$

Let $(L, A \cup B) \cup_{R}(M, A \cup C)=(N,(A \cup B))$ where $\forall \alpha \in(A \cup B) \cap(A \cup C)=$ $A \cup(B \cap C)=\left\{e_{1}, e_{2}, e_{3} \cdot e_{4}, e_{6}\right\}$,

$$
N(\alpha)=L(\alpha) \cup M(\alpha) \text {. }
$$

$$
=\left\{\begin{array}{l}
F(\alpha), \alpha \in(A-B) \cap(A-C)=A-(B \cup C)=\left\{e_{1}\right\} ; \\
F(\alpha) \cup G(\alpha), \alpha \in(A \cap B)-C=\left\{e_{2}\right\} ; \\
F(\alpha) \cup H(\alpha), \alpha \in(A \cap C)-B=\left\{e_{6}\right\} ; \\
G(\alpha) \cup H(\alpha), \alpha \in(B \cap C)-A=\left\{e_{4}\right\} ; \text { and } \\
F(\alpha) \cup\{G(\alpha) \cup H(\alpha)\}, \alpha \in A \cap B \cap C=\left\{e_{3}\right\} .
\end{array}\right.
$$

Thus $N\left(e_{1}\right)=F\left(e_{1}\right)=\left\{u_{1}, u_{2}, u_{3}, u_{4}\right\}$;

$$
\begin{aligned}
& N\left(e_{2}\right)=F\left(e_{2}\right) \cup\left(e_{2}\right)=\left\{u_{3}, u_{4}, u_{5}, u_{6}, u_{8}\right\} ; \\
& N\left(e_{3}\right)=F\left(e_{3}\right) \cup\left\{G\left(e_{3}\right) \cup H\left(e_{3}\right)\right\}=\left\{u_{1}, u_{2}, u_{3}, u_{4}, u_{7}, u_{8}\right\} ; \\
& N\left(e_{4}\right)=G\left(e_{4}\right) \cup H\left(e_{4}\right)=\left\{u_{4}, u_{5}, u_{6}, u_{7}, u_{8}\right\} ; \text { and } \\
& N\left(e_{6}\right)=F\left(e_{6}\right) \cup H\left(e_{6}\right)=\left\{u_{2}, u_{3}, u_{4}, u_{6}, u_{8}\right\} .
\end{aligned}
$$

It follows that $J(\alpha)$ and $N(\alpha)$ are not the same $\forall \alpha \in A \cup(B \cap C)$. Hence assertion (ii) holds.

Let us also illustrate (viii) with the following example using the soft sets in example 3.1.

Example 3.2: Let $(G, B) \quad(H, C)=(K, B \cap C)$ where $\forall \alpha \in B \cup C=\left\{e_{3}, e_{4}\right\}$,

It implies that

$$
\smile_{R} \quad K(\alpha)=G(\alpha)-H(\alpha) \text {. }
$$

$$
\begin{aligned}
& K\left(e_{3}\right)=G\left(e_{3}\right)-H\left(e_{3}\right)=\left\{u_{1}, u_{2}\right\} \\
& K\left(e_{4}\right)=G\left(e_{4}\right)-H\left(e_{4}\right)=\left\{u_{6}, u_{8}\right\} .
\end{aligned}
$$

Let $(F, A) \tilde{\cup}(K, B \cap C)=(J, A \cup(B \cap C))$ where $\forall \alpha \in A \cup(B \cap C)=\left\{e_{1}, e_{2}, e_{3}, e_{4}, e_{6}\right\}$,

We have,

$$
J(\alpha)=\left\{\begin{array}{l}
F(\alpha), \alpha \in A-(B \cap C)=\left\{e_{1}, e_{2}, e_{6}\right\} ; \\
F(\alpha), \alpha \in(B \cap C)-A=\left\{e_{4}\right\} ; \text { and } \\
F(\alpha) \cup K(\alpha), \alpha \in A(B \cap C)=\left\{e_{3}\right\} .
\end{array}\right.
$$

$$
\begin{array}{ll}
J\left(e_{1}\right)=\left\{u_{1}, u_{2}, u_{3}, u_{4}\right\} ; & J\left(e_{4}\right)=\left\{u_{6}, u_{8}\right\} ; \text { and } \\
J\left(e_{2}\right)=\left\{u_{2}, u_{4}, u_{5}, u_{6}\right\} ; & J\left(e_{6}\right)=\left\{u_{2}, u_{3}, u_{4}\right\} . \\
J\left(e_{3}\right)=\left\{u_{1}, u_{2}, u_{4}, u_{7}\right\} ; &
\end{array}
$$

Again, let $(F, A) \tilde{\cup}(G, B)=(L, A \cup B)$ where $\forall \alpha \in A \cup B=\left\{e_{1}, e_{2}, e_{3}, e_{4}, e_{5}, e_{6}\right\}$,

$$
L(\alpha)=\left\{\begin{array}{l}
F(\alpha), \alpha \in A-B=\left\{e_{1}, e_{6}\right\} ; \\
G(\alpha), \alpha \in B-A=\left\{e_{4}, e_{5}\right\} ; \text { and } \\
F(\alpha) \cup G(\alpha), \alpha \in A \cap B=\left\{e_{2}, e_{3}\right\} .
\end{array}\right.
$$

Then, 


$$
\begin{array}{ll}
L\left(e_{1}\right)=\left\{u_{1}, u_{2}, u_{3}, u_{4}\right\} ; & L\left(e_{4}\right)=\left\{u_{4}, u_{5}, u_{6}, u_{7}, u_{8}\right\} ; \\
L\left(e_{2}\right)=\left\{u_{3}, u_{4}, u_{5}, u_{6}, u_{8}\right\} ; & L\left(e_{5}\right)=\left\{u_{1}, u_{2}, u_{4}, u_{7}\right\} ; \text { and } \\
L\left(e_{3}\right)=\left\{u_{1}, u_{2}, u_{3}, u_{4}, u_{7}\right\} ; & L\left(e_{6}\right)=\left\{u_{2}, u_{3}, u_{4}\right\} .
\end{array}
$$

Let $(F, A) \tilde{\cup}(H, C)=(M, A \cup C)$ where $\forall \alpha \in A \cup C=\left\{e_{1}, e_{2}, e_{3}, e_{4}, e_{6}, e_{7}\right\}$,

$$
M(\alpha)=\left\{\begin{array}{l}
F(\alpha), \alpha \in A-C=\left\{e_{1}, e_{2}\right\} ; \\
H(\alpha), \alpha \in C-A=\left\{e_{4}, e_{7}\right\} ; \text { and } \\
F(\alpha) \cup H(\alpha), \alpha \in A \cap C=\left\{e_{3}, e_{6}\right\} .
\end{array}\right.
$$

$$
\begin{array}{ll}
M\left(e_{1}\right)=\left\{u_{1}, u_{2}, u_{3}, u_{4}\right\} ; & M\left(e_{4}\right)=\left\{u_{4}, u_{5}, u_{7}\right\} ; \\
M\left(e_{2}\right)=\left\{u_{3}, u_{4}, u_{5}, u_{6}\right\} ; & M\left(e_{6}\right)=\left\{u_{2}, u_{3}, u_{4}, u_{6}, u_{8}\right\} ; \\
M\left(e_{3}\right)=\left\{u_{1}, u_{2}, u_{3}, u_{4}, u_{7}, u_{8}\right\} ; \text { and } & M\left(e_{7}\right)=\left\{u_{2}, u_{3}, u_{5}, u_{7}\right\} .
\end{array}
$$

Let $(L, A \cup B) \smile_{R}(M, A \cup C)=(I,(A \cup B) \cap(A \cup C))=(I, A \cup(B \cap C))$ where

$\forall \alpha \in A \cup(B \cap C)=\left\{e_{1}, e_{2}, e_{3}, e_{4}, e_{6}\right\}$,

$$
\begin{aligned}
I(\alpha)=L(\alpha)-M(\alpha) \\
=\left\{\begin{array}{l}
F(\alpha)-F(\alpha)=\varnothing, \alpha \in A-(B \cup C)=\left\{e_{1}\right\} ; \\
G(\alpha)-F(\alpha), \alpha \in(A \cap B)-C=\left\{e_{2}\right\} ; \\
G(\alpha)-\{F(\alpha) \cup H(\alpha)\}, \alpha \in A \cap B \cap C=\left\{e_{3}\right\} ; \\
G(\alpha)-H(\alpha), \alpha \in(B \cap C)-A=\left\{e_{4}\right\} ; \text { and } \\
F(\alpha)-\{F(\alpha) \cup H(\alpha)\}, \alpha \in(A \cap C)-B=\left\{e_{6}\right\} .
\end{array}\right.
\end{aligned}
$$

Thus

$$
\begin{aligned}
& I\left(e_{1}\right)=\varnothing ; \\
& I\left(e_{2}\right)=\left\{u_{8}\right\} ; \\
& I\left(e_{3}\right)=\varnothing ; \\
& I\left(e_{4}\right)=\left\{u_{6}, u_{8}\right\} ; \text { and } \\
& I\left(e_{6}\right)=\varnothing .
\end{aligned}
$$

Hence $J(\alpha) \neq I(\alpha) \forall \alpha \in A \cup(B \cap C)=\left\{e_{1}, e_{2}, e_{3}, e_{4}, e_{6}\right\}$.

\section{Theorem 3.2 (Restricted Symmetric Difference Operation)}

Let $(F, A)$ and $(G, B)$ be two soft sets over the same universe $U$. then the following are satisfied.

$$
(F, A) \text { Ó }((G, B) \tilde{\Delta}(H, C))=((F, A) \text { Ó }(G, B)) \tilde{\Delta}((F, A) \text { Ó }(H, C))
$$

(ii) $\quad(F, A) \tilde{\Delta}(G, B)=(G, B) \tilde{\Delta}(F, A)$.

Proof: (i) Let

where $\forall \alpha \in B \cap C$,

$$
\begin{aligned}
(G, B) \tilde{\Delta}(H, C) & =(G, B) \cup_{R}(H, C) \quad(G, B) \mathrm{O}(H, C) \\
& =(K, B \cap C)_{R} \quad(\Psi R B \cap C) \\
& =(M, B \cap C)
\end{aligned}
$$

$K(\alpha)=G(\alpha) \cup H(\alpha)$;

$L(\alpha)=G(\alpha) \cap H(\alpha)$; and

$M(\alpha)=K(\alpha)-L(\alpha)$.

Let $(F, A) \mathrm{O}(M, B \cap C)=(N, A \cap(B \cap C))$ where $\forall \alpha \in A \cap(B \cap C)$, 


$$
\begin{aligned}
N(\alpha) & =F(\alpha) \cap M(\alpha) \\
& =F(\alpha) \cap[K(\alpha)-L(\alpha)] \\
& =F(\alpha) \cap\{[G(\alpha) \cup H(\alpha)]-[G(\alpha) \cap H(\alpha)]\} .
\end{aligned}
$$

Let $((F, A)$ Ó $(G, B)) \tilde{\Delta}((F, A)$ Ó $(H, C))$

$$
\begin{aligned}
& =(I, A \cap B) \tilde{\Delta}(J, A \cap C) \\
& =\left((I, A \cap B) \cup_{R}(J, A \cap C)\right) \quad((I, A \cap B) \mathrm{O}(J, A \cap C)) \\
& =(O,(A \cap B) \cap(A \cap C)) \quad(P,(A \cap B) \cap(A \cap C)) \\
& =(Q, A \cap B \cap C) \quad \smile_{R}
\end{aligned}
$$

where

$$
\begin{aligned}
\forall \alpha & \in A \cap B, I(\alpha)=F(\alpha) \cap G(\alpha) \\
\alpha & \in A \cap C, J(\alpha)=F(\alpha) \cap H(\alpha) .
\end{aligned}
$$

and $\forall a \in A \cap B \cap C$,

$$
\begin{aligned}
O(\alpha) & =I(\alpha) \cup J(\alpha) ; \\
P(\alpha) & =I(\alpha) \cap J(\alpha) ; \\
Q(\alpha) & =O(\alpha)-P(\alpha) \\
& =(I(\alpha) \cup J(\alpha))-(I(\alpha) \cap J(\alpha)) ; \\
& =\{[F(\alpha) \cap G(\alpha)] \cup[F(\alpha) \cap H(\alpha)]\} ; \\
& -\{[F(\alpha) \cap G(\alpha)] \cap[F(\alpha) \cap H(\alpha)]\} ; \text { and } \\
& =\mathrm{F}(\alpha) \cap\{[G(\alpha) \cup H(\alpha)]-[G(\alpha) \cap H(\alpha)]\} .
\end{aligned}
$$

Hence, $N(\alpha)$ and $Q(\alpha)$ are the same mappings $\forall \alpha \in A \cap B \cap C$, which completes the proof.

(iii) By Theorem 2.1 (i)

$$
(F, A) \tilde{\Delta}(G, B)=((F, A) \quad(G, B)) \cup_{R}((G, B) \quad(F, A))
$$

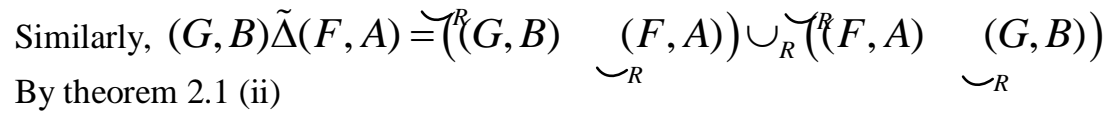

$$
\begin{aligned}
& ((F, A) \quad(G, B)) \cup_{R}((G, B) \quad(F, A)) \\
& =\left((G, B) \smile^{R}{ }_{R}(F, A)\right) \cup_{R}\left((F, \widehat{A})_{\cup_{R}^{R}}(G, B)\right)
\end{aligned}
$$

It implies $(F, A) \tilde{\Delta}(G, B)=(G, B) \tilde{\Delta}(F, A)$ proving (ii).

\section{Theorem 3.3 (Absorption Properties)}

Let $(F, A)$ and $(G, B)$ be two soft sets over the same universe $U$. Then we have the following:

(i) $\quad(F, A) \cup_{R}\left((F, A) \cap_{\varepsilon}(G, B)\right)=(F, A)$

(ii) $\quad(F, A) \mathrm{O}((F, A) \tilde{\cup}(G, B))=(F, A)$

(iii) $\quad(F, A) \cap_{\varepsilon}\left((F, A) \cup_{R}(G, B)\right)=(F, A)$

(iv) $\quad(F, A) \tilde{\cup}((F, A)$ Ó $(G, B))=(F, A)$

(v) $\quad(F, A) \cup_{R}((F, A)$ Ó $(G, B)) \tilde{\subset}(F, A)$

(vi) $\quad(F, A)$ Ó $\left((F, A) \cup_{R}(G, B)\right) \tilde{\subset}(F, A)$

(vii) $\quad(F, A) \tilde{\cup}\left((F, A) \cap_{\varepsilon}(G, B)\right) \tilde{\supset}(F, A)$, and

(viii) $\quad(F, A) \cap_{\varepsilon}((F, A) \tilde{\cup}(G, B)) \tilde{\supset}(F, A)$. 
Proof.

(i) Let $(F, A) \cup_{R}\left((F, A) \cap_{\varepsilon}(G, B)\right)$

$$
\begin{aligned}
& =(F, A) \cup_{R}(K, A \cup B) \\
& =(L, A \cap(A \cup B)) \\
& =(L, A)
\end{aligned}
$$

where $\forall \alpha \in A \cup B$,

$$
K(\alpha)=\left\{\begin{array}{l}
F(\alpha), \alpha \in A-B ; \\
G(\alpha), \alpha \in B-A ; \text { and } \\
F(\alpha) \cap G(\alpha), \alpha \in A \cap B .
\end{array}\right.
$$

and $\forall \alpha \in A$,

$$
\begin{aligned}
& L(\alpha)=F(\alpha) \cup K(\alpha) . \\
& \qquad(\alpha)=\left\{\begin{array}{l}
F(\alpha), \alpha \in A-B ; \\
F(\alpha) \cup G(\alpha), \alpha \in A \cap(B-A)=\varnothing ; \text { and } \\
F(\alpha) \cup\{F(\alpha) \cap G(\alpha)\}, \alpha \in A \cap B .
\end{array}\right. \\
& \quad=\left\{\begin{array}{l}
F(\alpha), \alpha \in A-B, \text { and } \\
F(\alpha), \alpha \in A \cap B .
\end{array}\right.
\end{aligned}
$$

Hence, $L(\alpha)=F(\alpha), \forall \alpha \in A$;

i.e., $(L, A)=(F, A)$ which proves (i).

Similarly (ii) can be proved.

(iii) Let $(F, A) \cap_{\varepsilon}\left((F, A) \cup_{R}(G, B)\right)$

$=(F, A) \cap_{\varepsilon}(K, A \cap B)$

$=(J, A \cup(A \cap B))=(J, A)$

where $\forall \alpha \in A \cap B$,

$$
K(\alpha)=F(\alpha) \cup G(\alpha)
$$

and $\forall \alpha \in A$,

$$
\begin{aligned}
J(\alpha) & =\left\{\begin{array}{l}
F(\alpha), \alpha \in A-(A \cap B) ; \\
K(\alpha), \alpha \in(A \cap B)-A=\varnothing ; \text { and } \\
F(\alpha) \cap K(\alpha)=F(\alpha) \cap\{F(\alpha) \cup G(\alpha)\}, \alpha \in A \cap(A \cap B) .
\end{array}\right. \\
& =\left\{\begin{array}{l}
F(\alpha), \alpha \in A-B ; \text { and } \\
F(\alpha), \alpha \in A \cap B .
\end{array}\right.
\end{aligned}
$$

Implying, $J(\alpha)=F(\alpha) \forall \alpha \in A$.

Hence $(J, A)=(F, A)$ proving (ii).

Similarly (iv) can be proved.

(v) Let $(F, A) \cup_{R}((F, A)$ Ó $(G, B))$

$$
\begin{aligned}
& =(F, A) \cup_{R}(K,(A \cap B)) \\
& =(P, A \cap(A \cap B)) \\
& =(P, A \cap B)
\end{aligned}
$$




$$
K(\alpha)=F(\alpha) \cap G(\alpha)
$$

where $\forall \alpha \in A \cap B, P(\alpha)=F(\alpha) \cup K(\alpha)$

$$
\begin{aligned}
& =F(\alpha) \cup\{F(\alpha) \cap G(\alpha)\} \\
& =F(\alpha) .
\end{aligned}
$$

Thus, $P(\alpha)=F(\alpha) \forall \alpha \in A \cap B \subset A$

Hence, $(P, A \cap B) \tilde{\subset}(F, A)$, which completes the proof.

Using similar arguments, (vi) can be proved.

$$
\text { Let } \begin{aligned}
& (F, A) \sim\left((F, A) \cap_{\varepsilon}(G, B)\right) \\
= & (F, A) \sim(K, A \cup B) \\
= & (O, A \cup(A \cup B)) \\
= & (O, A \cup B)
\end{aligned}
$$

where $\forall \alpha \in A \cup B$,

$$
K(\alpha)=\left\{\begin{array}{l}
F(\alpha), \alpha \in A-B \\
G(\alpha), \alpha \in B-A \\
F(\alpha) \cap G(\alpha), \alpha \in A \cap B
\end{array}\right.
$$

and

$$
\begin{aligned}
O(\alpha) & =\left\{\begin{array}{l}
F(\alpha), \alpha \in A-(A \cup B)=\varnothing ; \\
K(\alpha), \alpha \in(A \cup B)-A=B-A ; \text { and } \\
F(\alpha) \cup K(\alpha)=F(\alpha) \cup\{F(\alpha) \cap G(\alpha)=F(\alpha)\}, \alpha \in A \cap(A \cap B)=A ;
\end{array}\right. \\
& =\left\{\begin{array}{l}
G(\alpha), \alpha \in B-A ; \text { and } \\
F(\alpha), \alpha \in A .
\end{array}\right.
\end{aligned}
$$

It implies $O(\alpha)=F(\alpha) \forall \alpha \in A \subset A \cup B$.

Hence, $(O, A \cup B) \tilde{\supset}(F, A)$, which proves (vii).

Similarly, we can prove (viii).

Let us illustrate theorem 3.3 (i) in the following example.

\section{Example 3.3}

Let $U=\left\{u_{1}, u_{2}, u_{3}, u_{4}, u_{5}, u_{6}\right\}$ be a universe set consisting of six houses under consideration and let $E=\left\{e_{1}, e_{2}, e_{3}, e_{4}, u_{5}\right\}$ be the parameter with respect to $U$, where $e_{i}, i=1,2,3,4,5$ represent expensive, beautiful modern, 'in a good location', spacious, respectively.

Let $A$ and $B$ be subsets of $E$ given by $A=\left\{e_{2}, e_{3}, e_{5}\right\}$ and $B=\left\{e_{1}, e_{2}, e_{3}\right\}$.

Let $(F, A)$ and $(G, B)$ be two soft sets over the universe $U$ such that:

$$
\begin{aligned}
& (F, A)=\left\{\left(e_{2},\left\{u_{5}\right\}\right),\left(e_{3},\left\{u_{4}\right\}\right),\left(e_{5},\left\{u_{3}, u_{5}, u_{6}\right\}\right)\right\} ; \text { and } \\
& (G, B)=\left\{\left(e_{1},\left\{u_{1}, u_{2}, u_{6}\right\}\right),\left(e_{2},\left\{u_{3}, u_{4}, u_{5}\right\}\right),\left(e_{3},\left\{u_{2}, u_{3}, u_{4}\right\}\right)\right\} ;
\end{aligned}
$$

Let $(F, A) \cap_{\varepsilon}(G, B)=(K, A \cup B)$, where $\forall \alpha \in A \cup B=\left\{e_{1}, e_{2}, e_{3}, e_{5}\right\}$,

$$
K(\alpha)=\left\{\begin{array}{l}
F(\alpha), \alpha \in A-B=\left\{e_{5}\right\} \\
G(\alpha), \alpha \in B-A=\left\{e_{1}\right\} ; \text { and } \\
F(\alpha) \cap G(\alpha), \alpha \in A \cap B=\left\{e_{2}, e_{3}\right\}
\end{array}\right.
$$


Thus $(K, A \cup B)=\left\{\left(e_{1}\left\{u_{1}, u_{2}, u_{6}\right\}\right),\left(e_{2},\left\{u_{5}\right\}\right),\left(e_{3}\left\{u_{4}\right\}\right),\left(e_{5},\left\{u_{3}, u_{5}, u_{6}\right\}\right)\right\}$.

Let $(F, A) \cup_{R}(K, A \cup B)=(L, A \cap(A \cup B))=(L, A)$ where $\forall \alpha \in A=\left\{e_{2}, e_{3}, e_{5}\right\}$,

$$
\begin{aligned}
& L(\alpha)=F(\alpha) \cup K(\alpha) ; \\
& =\left\{\begin{array}{l}
F(\alpha), \alpha \in A-B=\left\{e_{5}\right\} ; \\
F(\alpha) \cup G(\alpha), \alpha \in \varnothing ; \text { and } \\
F(\alpha) \cup\{F(\alpha) \cap G(\alpha)\}, \alpha \in A \cap B=\left\{e_{2}, e_{3}\right\} .
\end{array}\right. \\
& =\left\{\begin{array}{l}
F(\alpha), \alpha \in\left\{e_{5}\right\} ; \text { and } \\
F(\alpha), \alpha \in\left\{e_{2}, e_{3}\right\} .
\end{array}\right.
\end{aligned}
$$

Hence,

$$
\begin{aligned}
(L, A) & =\left\{\left(e_{2},\left\{u_{5}\right\}\right),\left(e_{3},\left\{u_{4}\right\}\right),\left(e_{5},\left\{u_{3}, u_{5}, u_{6}\right\}\right)\right\} \\
& =(F, A), \text { establishing (i). }
\end{aligned}
$$

We illustrate (vi) of Theorem 3.3 in the following using soft sets in Example 3.3.

\section{Example 3.4}

Let $(F, A) \cup_{R}(G, B)=(K, A \cap B)$ where $\forall \alpha \in A \cap B=\left\{e_{2}, e_{3}\right\}$,

$$
K(\alpha)=F(\alpha) \cup G(\alpha) .
$$

Thus, $K\left(e_{2}\right)=F\left(e_{2}\right) \cup G\left(e_{2}\right)=\left\{u_{2}, u_{3}, u_{5}\right\}$; and

$$
K\left(e_{3}\right)=F\left(e_{3}\right) \cup G\left(e_{3}\right)=\left\{u_{2}, u_{3}, u_{4}\right\} .
$$

Let $(F, A) \quad(K, A \cap B)=(L, A \cap(A \cap B))=(L, A \cap B)$

where $\forall \alpha \in A \cap B=\left\{e_{2}, e_{3}\right\}$,

$$
L(\alpha)=F(\alpha) \cap K(\alpha) .
$$

Then $L\left(e_{2}\right)=F\left(e_{2}\right) \cap K\left(e_{2}\right)=\left\{u_{5}\right\}$ and $L\left(e_{3}\right)=F\left(e_{3}\right) \cap K\left(e_{3}\right)=\left\{u_{4}\right\}$.

Hence,

$$
\begin{aligned}
(L, A \cap B) & =\left\{\left(e_{2},\left\{u_{5}\right\}\right),\left(e_{3},\left\{u_{4}\right\}\right)\right\} \\
& \tilde{\subset}(F, A) \text { proving (vii). }
\end{aligned}
$$

Similarly, we can also illustrate (viii) in example 3.5, using the same argument in example 3.4 as follows:

\section{Example 3.5}

Let $(F, A) \tilde{\cup}(G, B)=(K, A \cup B)$ where $\forall \alpha \in A \cup B=\left\{e_{1}, e_{2}, e_{3}, e_{5}\right\}$,

$$
K(\alpha)=\left\{\begin{array}{l}
F(\alpha), \alpha \in A-B=\left\{e_{5}\right\} ; \\
G(\alpha), \alpha \in B-A=\left\{e_{1}\right\} ; \text { and } \\
F(\alpha) \cup G(\alpha), \alpha \in A \cap B=\left\{e_{2}, e_{3}\right\} .
\end{array}\right.
$$

Thus $K\left(e_{1}\right)=\left\{u_{1}, u_{2}, u_{6}\right\}$;

$$
\begin{aligned}
& K\left(e_{2}\right)=\left\{u_{2}, u_{3}, u_{5}\right\} ; \\
& K\left(e_{3}\right)=\left\{u_{2}, u_{3}, u_{4}\right\} ; \text { and } \\
& K\left(e_{5}\right)=\left\{u_{3}, u_{5}, u_{6}\right\} .
\end{aligned}
$$


Let $(F, A) \cap_{\varepsilon}(K, A \cup B)=(L, A \cup(A \cup B))=(L, A \cup B)$ where $\forall \alpha \in A \cup B=\left\{e_{1}, e_{2}, e_{3}, e_{5}\right\}$,

$$
L(\alpha)=\left\{\begin{array}{l}
F(\alpha), \alpha \in A-(A \cup B)=\varnothing ; \\
G(\alpha), \alpha \in(A \cup B)-A=B-A=\left\{e_{1}\right\} ; \text { and } \\
F(\alpha) \cap K(\alpha), \alpha \in A \cap(A \cup B)=A=\left\{e_{2}, e_{3}, e_{5}\right\} .
\end{array}\right.
$$

We have $L\left(e_{1}\right)=\left\{u_{1}, u_{2}, u_{6}\right\}$;

$$
\begin{aligned}
& L\left(e_{2}\right)=\left\{u_{5}\right\} ; \\
& L\left(e_{3}\right)=\left\{u_{4}\right\} ; \text { and } \\
& L\left(e_{5}\right)=\left\{u_{3}, u_{5}, u_{6}\right\} .
\end{aligned}
$$

Hence,

$$
\begin{aligned}
(L, A \cup B) & =\left\{\left(e_{1}\left\{u_{1}, u_{2}, u_{6}\right\}\right),\left(e_{2},\left\{u_{5}\right\}\right),\left(e_{3}\left\{u_{4}\right\}\right),\left\{u_{3}, u_{6}\right\}\right\} \\
& \tilde{\supset}(F, A) .
\end{aligned}
$$

Thus (viii) holds.

\section{Conclusion}

In this paper, we have proved and illustrated some new results pertaining to distributive and absorption properties in respect of various soft set operations described in the literature.

\section{References}

[1] A. Sezgin and A.O. Atagun, On Operations of Soft Set, Computers and Mathematics with Applications, 61 (2011), $1457-1467$.

[2] D. Molodtsov, Soft Set Theory - First Results, Computers and Mathematics with Applications, 37 (1999), 19-31.

[3] D. Pei and D. Miao, From Soft Sets to Information Systems, in Granular Computing, 2005 IEEE International Conference, Vol. 2 p. $617-621$.

[4] Fu Li, Notes on the Soft Operations, ARPN Journal of Systems and Software, Vol. 1 no. 6 (2011), $205-208$.

[5] K. Qin and Z. Hong, On Soft Equality, Journal of Computational and Applied Mathematics, 234 (2010), $1347-1355$.

[6] M.I. Ali, F. Feng, X. Liu, W.K. Min, M. Shabir, On Some New Operations on Soft Set Theory, Computers and Mathematics with Applications, 57 (2009), 1547-1553.

[7] P.K. Maji, R. Biswas, A.R. Roy, Soft Set Theory, Computers and Mathematics with Applications, 45 (2003), $555-562$. 